\title{
Current and State of Charge Estimation of Lithium-Ion Battery Packs Using Distributed Fractional Extended Kalman Filters
}

\author{
Martin Kupper ${ }^{1,2}$, Andreas Creutz ${ }^{1}$, Oliver Stark ${ }^{1}$, Stefan Krebs ${ }^{1}$ and Sören Hohmann ${ }^{1}$
}

\begin{abstract}
In this paper, a method for current and state of charge estimation of lithium-ion battery packs is proposed. On the basis of a fractional 1-RQ equivalent circuit cell model, a string model containing cells in serial connection, and a pack model containing strings in parallel connection is built up. In order to reduce computational costs, the model is distributed string-wise into subsystems. An algorithm using distributed fractional extended Kalman filters is applied to estimate the state of charge of all cells of each string, locally. To avoid costly measurements of numerous currents, a model based calculation is proposed which describes how the total battery current is split up between the strings. The algorithm is tested and validated using measurement data.
\end{abstract}

\section{INTRODUCTION}

Lithium-ion batteries ensure the power supply of numerous applications, such as consumer electronics and electric vehicles [1]-[3]. One of their most important characteristic is the state of charge (SOC). It provides information about the remaining utilization capability of a battery-powered device. Therefore, exact knowledge of the SOC allows to exploit the full capacity of the battery without approaching dangerous situations like deep discharge or overcharge [1], [4].

The SOC is defined in [5] as the amount of remaining electric charge in the battery $Q(t)$ in reference to its rated electric charge $Q_{n}$ by

$$
\operatorname{SOC}(t):=\frac{Q(t)}{Q_{n}}
$$

A large number of methods has evolved in order to estimate the SOC of a lithium-ion cell, since it cannot be measured directly [5], [6]. The two most basic approaches are Coulomb counting (CC) and the open circuit voltage (OCV) methods. The first one relies on precise measurement, parameterization of loss mechanisms, and initialization [6], [7] and is therefore only short-term accurate. The latter takes advantage of the OCV-SOC relationship (Fig. 1), but requires long rest times of the battery without load. Moreover, the OCV-SOC curve often has a hysteresis property dependent on being in a charging or discharging cycle [1], [6].

Advanced methods combine both approaches using a battery model and a state estimation algorithm. White box approaches such as electrochemical models are in most cases too complex and, therefore, have to be simplified [8]. Black box approaches like neural networks are difficult to interpret

\footnotetext{
*This work was supported by the ITK Engineering GmbH

${ }^{1}$ Martin Kupper, Andreas Creutz, Oliver Stark, Stefan Krebs and Sören Hohmann are with the Institute of Control Systems, Karlsruhe Institute of Technology, Karlsruhe, Germany martin.kupper@kit.edu

${ }^{2}$ Martin Kupper is also with the ITK Engineering GmbH, Rülzheim, Germany
}

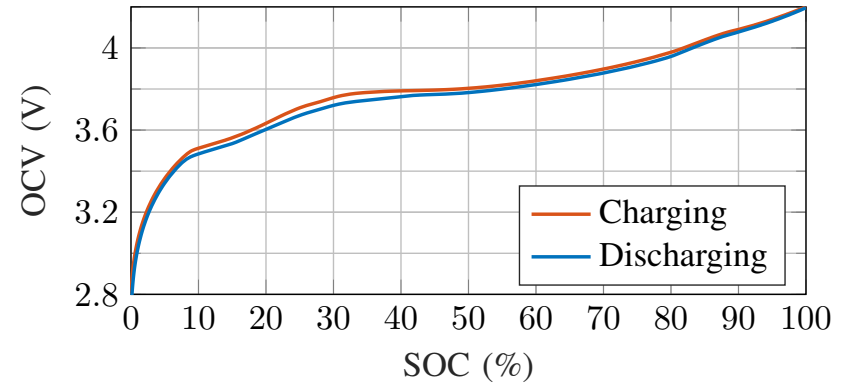

Fig. 1. OCV-SOC characteristics of a KOKAM cell of type SLPB834374H.

and reliant on training data [9]. A gray box technique to describe lithium-ion battery cells make use of fractional order models. Fractional calculus can be used to simplify electrochemical models with a reduced number of parameters [10]. Moreover, even simple fractional equivalent circuit models offer a good approximation of the battery behaviour, requiring fewer parameters than integer order models [11]. Therefore, fractional models offer a good basis for SOC estimation and are focus of research [12]-[15].

Many methods in the literature aim to estimate the SOC of single lithium-ion cells. However, applications like electric vehicles require the energy of numerous battery cells. Therefore, these cells are operated in serial and parallel as battery packs. As listed in [16], there is a variety of estimation approaches. Many algorithms estimate a single SOC of the battery pack, such as [2], [17]-[20]. There, an equivalent circuit model (ECM) is used to model the pack as a whole and an estimation based on Kalman filtering is applied. However, as discussed in [21], it is difficult to define a meaningful SOC for the whole pack. Furthermore, variations between cells as a result of manufacturing tolerances, different serial resistances, or temperature differences are inevitable [22]. These variations lead to unequal degradation rates of the cells [23] and to an uneven current distribution of battery strings in parallel connection as discussed in [22], [24], [25].

As a result, the SOC of each cell should be estimated to assure safe and efficient operation [26]. One approach to save computational costs is the estimation of an average SOC for serially connected cells which is then individually corrected for each cell, as proposed in [21], [22]. On the other hand, the current of each string has to be measured separately, which is costly and causes power losses in the system [27], especially when facing numerous strings in large battery packs. In [28], an iterative algorithm for the simulation of the current distribution is presented. However, 
it requires a lot of computational power. Another approach has been given in [27] which employs a filtered terminal voltage for SOC estimation without current measurement, but modeling and measurement errors are not considered. In [29], a real-time capable approach is given which estimates the string currents as well as the SOCs of all cells in a battery using cascaded fractional models. However, since an unidirectional communication procedure between the strings is used, an error propagation to all following subsystems is likely possible. An algorithm where each subsystem receives the same information is expected to be more robust, but the mesh current based model of [29] is not suitable for this. Summarizing, except [29], there exist no real-time capable, model based methods for current estimation of battery packs, yet. However, there exists an approach for distributed estimation of the temperature field across a Liion battery [30] showing that a distribution scheme achieves reduced computational costs.

In this paper, we derive a fractional order battery pack model from an equivalent circuit cell model. This pack model is distributed string-wise into subsystems to enable an order reduction in comparison to the pack model. For each subsystem a fractional extended Kalman filter is employed for online and local SOC estimation of all cells in the string. Due to the reduced order, and based on the fact that all string models are structurally identical, this approach provides scalability and reduced computational costs. Applying Kirchhoff's law, a model based calculation of the string currents is introduced. This calculation is used to enable a string current estimation which requires measurements of the total current or of the total voltage, only. Accordingly, string current measurements are not needed. Depending on if the total current or the total voltage is measured, an information exchange between all subsystems is required or not. Although the proposed approach is derived on the basis of fractional order models in this paper, it is also applicable to integer-order models.

\section{FRACTIONAL ORDER SYSTEMS}

There are several definitions of fractional order derivatives. One of the most commonly used is the definition of Grünwald-Letnikov. A discrete-time approximation of the derivative of fractional order of a function $\boldsymbol{f}$ is given in [31], [32], here generalized for multidimensional functions by

$$
{ }_{0} \mathrm{D}_{t}^{\boldsymbol{\alpha}_{k}} \boldsymbol{f}(t) \approx \boldsymbol{T}_{\boldsymbol{\alpha}_{k}}^{-1} \sum_{j=0}^{k+1}(-1)^{j} \boldsymbol{\Upsilon}_{\boldsymbol{\alpha}_{k}, j} \boldsymbol{f}((k+1-j) T)
$$

where 0 is the lower limit of the differentiation. The individual fractional derivatives and orders, respectively, are denoted by ${ }_{0} \mathrm{D}_{t}^{\boldsymbol{\alpha}_{k}} \boldsymbol{f}(t):=\left[{ }_{0} \mathrm{D}_{t}^{\alpha_{1, k}} f_{1}(t), \ldots,{ }_{0} \mathrm{D}_{t}^{\alpha_{n, k}} f_{n}(t)\right]^{T}$ with an extension of the binomial coefficients

$$
\boldsymbol{\Upsilon}_{\boldsymbol{\alpha}_{k}, j}:=\operatorname{diag}\left\{\left(\begin{array}{c}
\alpha_{1, k} \\
j
\end{array}\right), \ldots,\left(\begin{array}{c}
\alpha_{n, k} \\
j
\end{array}\right)\right\}
$$

and the weighted sampling times

$$
\boldsymbol{T}_{\boldsymbol{\alpha}_{k}}:=\operatorname{diag}\left\{T^{\alpha_{1, k}}, \ldots, T^{\alpha_{n, k}}\right\} .
$$

\section{A. Short Memory Principle}

In order to evaluate the derivative at time $t=k T$ in (2), more and more past function values of $\boldsymbol{f}$ are needed as time increases. However, the elements of $\boldsymbol{\Upsilon}_{\boldsymbol{\alpha}_{k}, j}$ converge to zero for $k \rightarrow \infty$, making older values of $\boldsymbol{f}$ less important for the calculation of the derivative. Therefore, only a fixed number $L$ of past values is considered which is called short memory principle (SMP) [31], [33].

\section{B. Initialization}

A fractional derivative is not a local operator and, therefore, dependent on its history [34]. Hence, an initialization function has to be taken into account. It describes the influence of the history of $\boldsymbol{f}(t)$ before the lower limit $t=0$ of (2) on the function at the current time $t$. The influence of an initialization function for state estimation of battery cells with a fractional extended Kalman filter was examined in [15]. The impact of the uncertainty of the filter by its error covariance matrix is larger than the influence of an initialization function. In addition, the initialization function is usually unknown in practice and it also loses influence during the estimation process due to the SMP and progressing time as discussed in II-A. Therefore, initialization functions will be neglected in this paper.

\section{Fractional Order State Space Representation}

A discrete-time fractional order state space model is given in [29] by

$$
\begin{aligned}
\boldsymbol{x}_{k+1} & =\boldsymbol{f}_{k}\left(\boldsymbol{x}_{k}, \boldsymbol{u}_{k}\right)-\sum_{j=1}^{k+1}(-1)^{j} \boldsymbol{\Upsilon}_{\boldsymbol{\alpha}_{k}, j} \boldsymbol{x}_{k+1-j}+\boldsymbol{v}_{k} \\
\boldsymbol{y}_{k} & =\boldsymbol{g}_{k}\left(\boldsymbol{x}_{k}, \boldsymbol{u}_{k}\right)+\boldsymbol{w}_{k} .
\end{aligned}
$$

where $\boldsymbol{x}_{k} \in \mathbb{R}^{n}$ is the state, $\boldsymbol{u}_{k} \in \mathbb{R}^{p}$ the input, $\boldsymbol{v}_{k} \in$ $\mathbb{R}^{n}$ the system noise, $\boldsymbol{y}_{k} \in \mathbb{R}^{q}$ the output, and $\boldsymbol{w}_{k} \in \mathbb{R}^{q}$ the measurement noise [15], [31]. The fractional derivatives of orders $\boldsymbol{\alpha}_{k}$ are implicitly contained in $\boldsymbol{\Upsilon}_{\boldsymbol{\alpha}_{k}, j}$ which is induced by the Grünwald-Letnikov definition in (2). Note that this model is not a state space model in its classical sense, since the system behaviour is additionally dependent on past states as discussed in II-A, II-B, and further in [34]. Therefore, it is commonly called pseudo state space model. For simplicity reasons, the usual description as state space model will nevertheless be used in the following.

\section{BATTERY MODEL}

\section{A. Model of a Battery Cell}

Fractional order battery models can be deduced from a physical analysis of the cell, making their parameters interpretable [35]. Furthermore, they are able to provide better approximations of the battery impedance with the same or a lower number of parameters than integer order models [15]. A simple but effective way of modeling battery cells is a 1-RQ equivalent circuit model (Fig. 2). The model consists of an internal resistance $R_{i}$, a RQ circuit with resistance $R$ and a fractional capacitor $Q$ of order $\alpha$, and a nonlinear capacitance $C$. The voltage-charge characteristic of $C$ is 


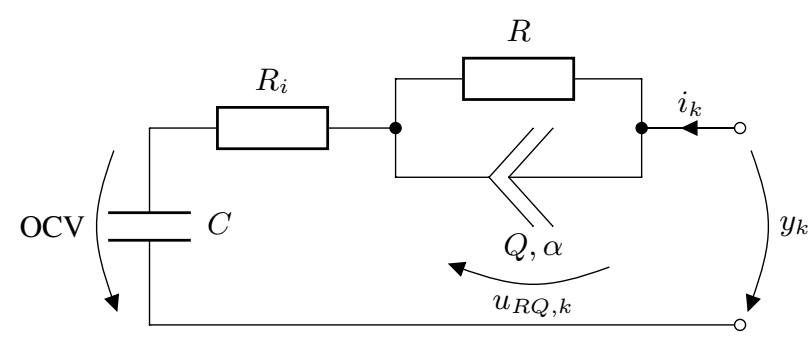

Fig. 2. 1-RQ equivalent circuit model of a battery cell.

interpolated by a polynomial and described by the averaged OCV-SOC relations of Fig. 1, since the hysteresis can be neglected for many mid and high temperature applications [6]. Since the impedance of the cell is dependent on the SOC, all parameters $R_{i}, R, Q$ and $\alpha$ of the model are also dependent on the SOC. They are interpolated using a shapepreserving piecewise cubic function. E.g., the order $\alpha$ varies between 0.5 and 0.75 subject to the SOC. The model of the single cell (upper index $c$ ) is given in [15] by

$$
\begin{aligned}
\boldsymbol{x}_{k+1}^{c}:= & \left(\begin{array}{c}
\mathrm{SOC}_{k+1} \\
u_{R Q, k+1}
\end{array}\right)=\left(\begin{array}{c}
\frac{100 T \eta i_{k}}{Q_{n}} \\
\frac{T^{\alpha}}{Q}\left(i_{k}-\frac{u_{R Q, k}}{R}\right)
\end{array}\right) \\
& -\sum_{j=1}^{k+1}(-1)^{j} \boldsymbol{\Upsilon}_{\boldsymbol{\alpha}, j}\left(\begin{array}{c}
\mathrm{SOC}_{k+1-j} \\
u_{R Q, k+1-j}
\end{array}\right)+\left(\begin{array}{c}
v_{S O C, k} \\
v_{R Q, k}
\end{array}\right) \\
= & : \boldsymbol{f}_{k}^{c}\left(\boldsymbol{x}_{k}^{c}, i_{k}\right)+\boldsymbol{v}_{k}^{c} \\
y_{k}^{c}:= & \mathrm{OCV}+u_{R Q, k}+R_{i} i_{k}+w_{k} \\
= & : \boldsymbol{g}_{k}^{c}\left(\boldsymbol{x}_{k}^{c}, i_{k}\right)+w_{k}^{c}
\end{aligned}
$$

where the cell current $i_{k}$ is the input, $u_{R Q, k}$ is the voltage of the RQ circuit pursuant to Fig. 2, $\eta$ is the coulomb efficiency, $T$ is the sampling time, the states of the cell are $\boldsymbol{x}_{k}^{c}$, the cell voltage $y_{k}^{c}$ is the output, and $\boldsymbol{v}_{k}^{c}:=\left(v_{S O C, k}, v_{R Q, k}\right)^{\top}$ and $w_{k}^{c}:=w_{k}$ are additive white gaussian noises which describe model uncertainties and measurement noise, respectively.

\section{B. Model of a Battery String}

A serial connection of $S$ single cells is called a battery string. As the cells are serially connected, the cell currents are identical in every cell. The model of a single string (upper index $s$ ) results cell-wise as

$$
\begin{aligned}
\boldsymbol{x}_{k+1}^{s} & :=\left(\begin{array}{c}
\boldsymbol{x}_{1, k+1}^{c} \\
\vdots \\
\boldsymbol{x}_{S, k+1}^{c}
\end{array}\right)=\left(\begin{array}{c}
\boldsymbol{f}_{1, k}^{c}\left(\boldsymbol{x}_{1, k}^{c}, i_{k}\right) \\
\vdots \\
\boldsymbol{f}_{S, k}^{c}\left(\boldsymbol{x}_{S, k}^{c}, i_{k}\right)
\end{array}\right)+\left(\begin{array}{c}
\boldsymbol{v}_{1, k}^{c} \\
\vdots \\
\boldsymbol{v}_{S, k}^{c}
\end{array}\right) \\
& =: \boldsymbol{f}_{k}^{s}\left(\boldsymbol{x}_{k}^{s}, i_{k}\right)+\boldsymbol{v}_{k}^{s} \\
\boldsymbol{y}_{k}^{s} & :=\left(\begin{array}{c}
\boldsymbol{y}_{1, k+1}^{c} \\
\vdots \\
\boldsymbol{y}_{S, k+1}^{c}
\end{array}\right)=\left(\begin{array}{c}
\boldsymbol{g}_{1, k}^{c}\left(\boldsymbol{x}_{1, k}^{c}, i_{k}\right) \\
\vdots \\
\boldsymbol{g}_{S, k}^{c}\left(\boldsymbol{x}_{S, k}^{c}, i_{k}\right)
\end{array}\right)+\left(\begin{array}{c}
w_{1, k}^{c} \\
\vdots \\
w_{S, k}^{c}
\end{array}\right) \\
= & \boldsymbol{g}_{k}^{s}\left(\boldsymbol{x}_{k}^{s}, i_{k}\right)+\boldsymbol{w}_{k}^{s}
\end{aligned}
$$

with $\boldsymbol{v}_{k}^{s}:=\left(\boldsymbol{v}_{1, k}^{c}, \ldots, \boldsymbol{v}_{S, k}^{c}\right)^{\top}$ and $\boldsymbol{w}_{k}^{s}:=\left(w_{1, k}^{c}, \ldots, w_{S, k}^{c}\right)^{\top}$ as abbreviations for the noises. The functions $\boldsymbol{f}_{k}^{s}\left(\boldsymbol{x}_{k}^{s}, i_{k}\right)$ and $\boldsymbol{g}_{k}^{s}\left(\boldsymbol{x}_{k}^{s}, i_{k}\right)$ denote the system and output equations of the whole string. Note that $f^{s}$ comprises the dynamics of each cell (7) which are of varying fractional orders.

\section{Model of a Battery Pack}

In order to model a battery pack, $P$ parallel strings, with $S$ cells per string are assumed (Fig. 3). The resulting state vector $\boldsymbol{x}_{k}^{b}$ is assembled string-wise. The output vector $\boldsymbol{y}_{k}^{b}$ is arranged in the same way and the input vector is built up of the string currents $\boldsymbol{i}_{k}^{b}:=\left(i_{1, k}, \ldots, i_{P, k}\right)^{\top}$. The result is a state space model of the entire battery pack (upper index $b$ ), identical to [29] as

$$
\begin{aligned}
\boldsymbol{x}_{k+1}^{b} & =\left(\begin{array}{c}
\boldsymbol{x}_{1, k+1}^{s} \\
\vdots \\
\boldsymbol{x}_{P, k+1}^{s}
\end{array}\right)=\left(\begin{array}{c}
\boldsymbol{f}_{1, k}^{s}\left(\boldsymbol{x}_{1, k}^{s}, i_{1, k}\right) \\
\vdots \\
\boldsymbol{f}_{P, k}^{s}\left(\boldsymbol{x}_{P, k}^{s}, i_{P, k}\right)
\end{array}\right)+\left(\begin{array}{c}
\boldsymbol{v}_{1, k}^{s} \\
\vdots \\
\boldsymbol{v}_{P, k}^{s}
\end{array}\right) \\
& =: \boldsymbol{f}_{k}^{b}\left(\boldsymbol{x}_{k}^{b}, \boldsymbol{i}_{k}^{b}\right)+\boldsymbol{v}_{k}^{b} \\
\boldsymbol{y}_{k}^{b} & =\left(\begin{array}{c}
\boldsymbol{y}_{1, k}^{s} \\
\vdots \\
\boldsymbol{y}_{P, k}^{s}
\end{array}\right)=\left(\begin{array}{c}
\boldsymbol{g}_{1, k}^{s}\left(\boldsymbol{x}_{1, k}^{s}, i_{1, k}\right) \\
\vdots \\
\boldsymbol{g}_{P, k}^{s}\left(\boldsymbol{x}_{P, k}^{s}, i_{P, k}\right)
\end{array}\right)+\left(\begin{array}{c}
\boldsymbol{w}_{1, k}^{s} \\
\vdots \\
\boldsymbol{w}_{P, k}^{s}
\end{array}\right) \\
& =: \boldsymbol{g}_{k}^{b}\left(\boldsymbol{x}_{k}^{b}, \boldsymbol{i}_{k}^{b}\right)+\boldsymbol{w}_{k}^{b}
\end{aligned}
$$

with $\quad \boldsymbol{v}_{k}^{b}:=\left(\boldsymbol{v}_{1, k}^{s}, \ldots, \boldsymbol{v}_{P, k}^{s}\right)^{\top} \quad$ and $\quad \boldsymbol{w}_{k}^{b} \quad:=$ $\left(\boldsymbol{w}_{1, k}^{s}, \ldots, \boldsymbol{w}_{P, k}^{s}\right)^{\top}$ being abbreviations for the noises. Note that the functions $\boldsymbol{f}^{c}, \boldsymbol{f}^{s}$ and $\boldsymbol{f}^{b}$ include implicitly the sum induced by the Grünwald-Letnikov definition and are, therefore, of fractional order. The equations (7) - (18) are of the structure of (5) and (6), respectively. To avoid all possibilities of confusion, the inputs $\boldsymbol{u}_{k}$ of (5) and (6) are no voltages but the string currents.

\section{Model Based Current Calculation}

As stated in the introduction, current measurements of each string in large battery packs can be costly and cause

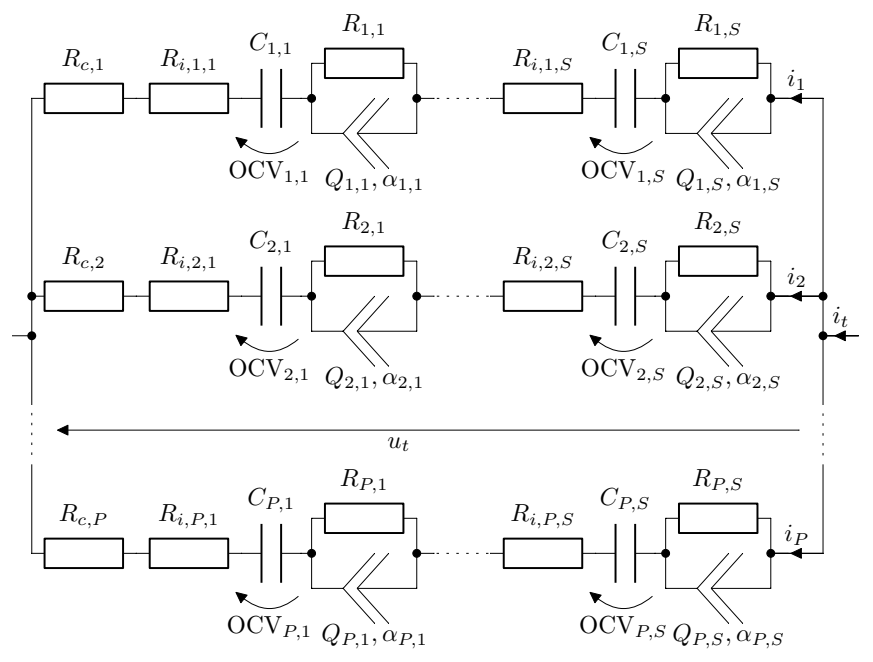

Fig. 3. Model of the battery pack. 
power losses. Therefore, the distribution of the currents is calculated by a model based approach in this paper which is described in this section. Since the approach only considers algebraic equations, the time-index $k$ is omitted in this section for increased readability.

Theorem 1 (Current Distribution). The current distribution can be calculated as

$$
\begin{aligned}
i_{p} & =i_{t} \frac{R_{i, t}}{R_{i, p}}+\frac{R_{i, t}}{R_{i, p}}\left(\sum_{a=1}^{P} \frac{u_{m, a}-u_{m, p}}{R_{i, a}}\right) \\
& =\frac{u_{t}-u_{m, p}}{R_{i, p}}
\end{aligned}
$$

whereby the indices $(*)_{p}$ and $(*)_{a}$ refer to the variables of the $p$-th or a-th string, respectively. The index $(*)_{t}$ refers to the total battery pack. The variable

$$
R_{i, p}:=\sum_{s=1}^{S} R_{i, p, s}+R_{c, p}
$$

describes the sum of the inner resistances of all cells of string $p$, also considering additional wire and connector resistances $R_{c, p}$. The index $(*)_{p, s}$ refers to the $s$-th cell in the $p$-th string. The variable

$$
R_{i, t}:=\left(\sum_{a=1}^{P} \frac{1}{R_{i, a}}\right)^{-1}
$$

can be interpreted as total resistance, and

$$
i_{t}:=\sum_{a=1}^{P} i_{a}
$$

is the total battery current according to Kirchhoff's current law. The auxiliary voltages

$$
u_{m, p}:=\sum_{s=1}^{S}\left(O C V_{p, s}+u_{R Q, p, s}\right)
$$

describe the sum of $O C V$ and $u_{R Q}$ for the cells of a single string $p$.

Proof. Applying Kirchhoff's voltage law, the total voltage $u_{t}$ equals the voltage of each string $u_{p}$

$$
u_{t}=u_{p} \quad \forall p
$$

The voltage of a string is the sum of the voltages of all $S$ cells of the string as well as the voltage over the resistance $R_{c, p}$

$$
u_{p}=\sum_{s=1}^{S}\left(\mathrm{OCV}_{p, s}+u_{R Q, p, s}+R_{i, p, s} i_{p}\right)+R_{c, p} i_{p} .
$$

Using (21), (24), and (25), equation (26) is simplified to

$$
u_{t}=u_{m, p}+i_{p} R_{i, p} \quad \forall p .
$$

Solving (27) for $i_{p}$, and inserting in (23) results in

$$
i_{t}=\sum_{a=1}^{P} i_{a}=\sum_{a=1}^{P} \frac{u_{t}-u_{m, a}}{R_{i, a}}=u_{t} \sum_{a=1}^{P} \frac{1}{R_{i, a}}-\sum_{a=1}^{P} \frac{u_{m, a}}{R_{i, a}} .
$$

Rearranging the equation for $u_{t}$ and using (22) yields

$$
u_{t}=\left(i_{t}+\sum_{a=1}^{P} \frac{u_{m, a}}{R_{i, a}}\right) R_{i, t} .
$$

With (29), equation (27) can be reformulated

$$
\begin{aligned}
i_{p} & =\frac{u_{t}-u_{m, p}}{R_{i, p}} \\
& =i_{t} \frac{R_{i, t}}{R_{i, p}}+\frac{R_{i, t}}{R_{i, p}}\left(\sum_{a=1}^{P} \frac{u_{m, a}-u_{m, p}}{R_{i, a}}\right) .
\end{aligned}
$$

which leads to (19) and (20) and concludes the proof. Hereby, $a$ is the index of summation over all strings and $p$ is used to describe which string is considered.

As a result, it can be seen that the string current calculation in (19) is split into two parts. On the one hand, the total current is divided into the strings by means of the ratio between total resistance and resistance of the particular string $p$. On the other hand, there are compensating currents between the strings, dependent on the difference between the state-related voltages OCV and $u_{R Q}$ of string $p$ and all other strings. These compensating currents are also dependent on the ratio between total resistance and resistance of string $p$.

Alternatively, the string currents can be calculated using (20) which requires knowledge about the total voltage instead of the total current. An advantage of this method is that the current of string $p$ is only dependent on the resistances and the state-related voltages $\mathrm{OCV}$ and $u_{R Q}$ of its own string $p$. Hence, for the calculation of the current in string $p$ no information about other strings are needed, in contrast to the previous case.

We assume in the rest of the paper that either the total voltage or the total current of the battery pack is being measured.

\section{E. Distribution of the Battery Pack Model}

The pack model is divided into a coupled set of subsystems in this paper to achieve an order reduction. Since the current as input variable is equal within one string, a string-wise distribution of the pack (see (11) - (14)) is preferable. Accordingly, states, outputs, and input of each subsystem correspond to states, outputs, and input of one string. The same applies to the system and output equations, and the noises. Therefore, the order of the subsystem in comparison to the total battery pack model is reduced by $2 \cdot S /(2 \cdot S \cdot P)=$ $1 / P$. The complexity of a centralized Kalman filter algorithm $\mathcal{O}\left((2 \cdot S \cdot P)^{3}\right)$ is hence reduced to $\mathcal{O}\left((2 \cdot S)^{3}\right)$ for the distributed Kalman filters [30]. Note that other choices for the subsystems are also possible, e.g., cell-wise subsystems.

\section{ESTIMATION PROCEDURE}

In this section, the model based SOC and current estimation procedure for the battery pack is presented. A distributed fractional extended Kalman filter (DFEKF) which is based on [15], [36] is used for local estimation of each subsystem.

Case 1: (Total current is measured): For the model based string current calculation of (19), information from other 
subsystems about their states and resistances is required. Since the true values are not available, the subsystems have to exchange their estimations in order to calculate the string currents. Furthermore, an all-to-all connection between the subsystems is required, because the current calculation needs information from all subsystems as seen in (19). Such an information exchange can be implemented by a central fusion node as shown in Fig. 4, or separately in each subsystem for increased robustness. Because the dependency between the subsystems is already included in the current distribution model, the fusion step of a distributed state estimation algorithm as, e.g., in [36] can be replaced by the current calculation (19).

Case 2: (Total voltage is measured): For a current calculation as in (20), an information exchange between subsystems is not necessary. Only the measured total voltage must be available at all subsystems.

Both cases, (19) and (20) are algebraic equations, because $i_{p, k}$ is dependent on variables of the same time-step $k$, only. The corrected states $\hat{\boldsymbol{x}}_{a, k \mid k}\left(\hat{i}_{a, k}\right)$ are dependent on the current of the same time-step $k$ and, therefore, can not be used for the current calculation. Hence, for the current estimation, the predicted states $\hat{\boldsymbol{x}}_{a, k \mid k-1}\left(\hat{i}_{a, k-1}\right)$ must be used, because they depend only on the current of the previous time-step $k-1$. As a result, a reasonable sequence for the estimation procedure is to calculate the current estimation and the state correction for the current time-step $k$ first, and then the state prediction for the next time-step $k+1$, as shown in Fig. 4 for case 1 . It follows:

Current estimation (case 1):

$\hat{i}_{p, k}=i_{t, k} \frac{R_{i, t, k}}{R_{i, p, k}}+\frac{R_{i, t, k}}{R_{i, p, k}}\left(\sum_{a=1}^{P} \frac{\hat{u}_{m, a, k \mid k-1}-\hat{u}_{m, p, k \mid k-1}}{R_{i, a, k}}\right)$

or

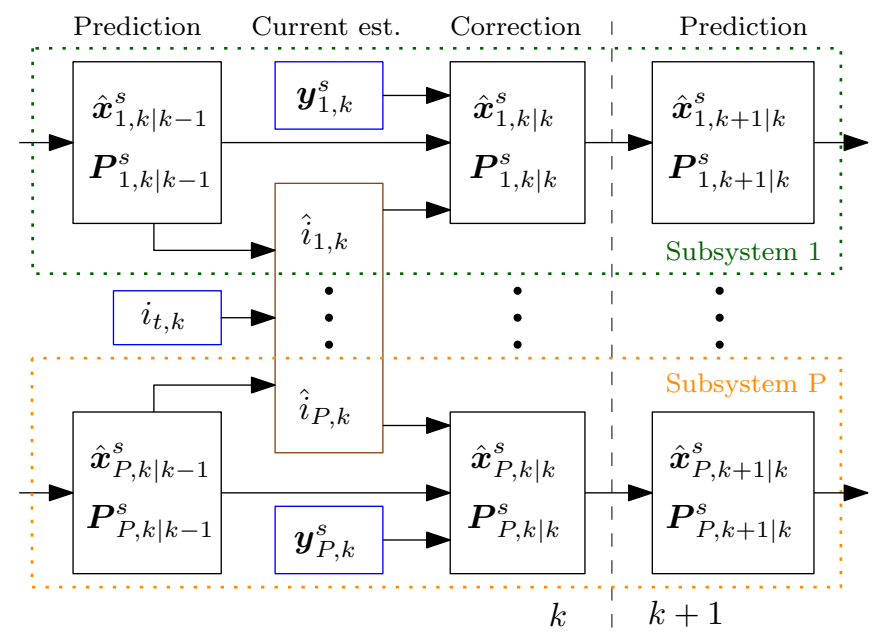

Fig. 4. Processing order and communication between the Kalman filters using a central node (brown) for data fusion in case of using the total current measurement (19). Measurements are highlighted in blue.
Current estimation (case 2):

$$
\hat{i}_{p, k}=\frac{u_{t, k}-\hat{u}_{m, p, k \mid k-1}}{R_{i, p, k}}
$$

State correction:

$$
\begin{aligned}
\boldsymbol{K}_{p, k}^{s}= & \boldsymbol{P}_{p, k \mid k-1}^{s} \boldsymbol{G}_{p, k}^{s, \top} \\
& \cdot\left(\boldsymbol{G}_{p, k}^{s} \boldsymbol{P}_{p, k \mid k-1}^{s} \boldsymbol{G}_{p, k}^{s, \top}+\boldsymbol{R}_{p, k}^{s}\right)^{-1} \\
\hat{\boldsymbol{x}}_{p, k \mid k}^{s}= & \hat{\boldsymbol{x}}_{p, k \mid k-1}^{s}+\boldsymbol{K}_{p, k}^{s}\left[\boldsymbol{y}_{p, k}^{s}-\boldsymbol{g}_{p, k}^{s}\left(\hat{\boldsymbol{x}}_{p, k \mid k-1}^{s}, \hat{i}_{p, k}\right)\right] \\
\boldsymbol{P}_{p, k \mid k}^{s}= & \left(\boldsymbol{I}-\boldsymbol{K}_{p, k}^{s} \boldsymbol{G}_{p, k}^{s}\right) \boldsymbol{P}_{p, k \mid k-1}^{s}\left(\boldsymbol{I}-\boldsymbol{K}_{p, k}^{s} \boldsymbol{G}_{p, k}^{s}\right)^{\top} \\
& +\boldsymbol{K}_{p, k}^{s} \boldsymbol{R}_{p, k}^{s} \boldsymbol{K}_{p, k}^{s, \top}
\end{aligned}
$$

State prediction:

$$
\begin{aligned}
\hat{\boldsymbol{x}}_{p, k+1 \mid k}^{s}= & \boldsymbol{f}_{p, k}^{s}\left(\hat{\boldsymbol{x}}_{p, k \mid k}^{s}, \hat{i}_{p, k}\right) \\
\boldsymbol{P}_{p, k+1 \mid k}^{s}= & \left(\boldsymbol{F}_{p, k}^{s}+\boldsymbol{\Upsilon}_{p, \boldsymbol{\alpha}_{k}, 1}^{s}\right) \boldsymbol{P}_{p, k \mid k}^{s}\left(\boldsymbol{F}_{p, k}^{s, \top}+\boldsymbol{\Upsilon}_{p, \boldsymbol{\alpha}_{k}, 1}^{s, \top}\right) \\
& +\boldsymbol{Q}_{p, k}^{s}+\sum_{j=2}^{L+1} \boldsymbol{\Upsilon}_{p, \boldsymbol{\alpha}_{k}, j}^{s} \boldsymbol{P}_{p, k+1-j \mid k+1-j}^{s} \boldsymbol{\Upsilon}_{p, \boldsymbol{\alpha}_{k}, j}^{s, \top}
\end{aligned}
$$

with the auxiliary voltage

$$
\hat{u}_{m, a, k \mid k-1}:=\sum_{s=1}^{S} \operatorname{OCV}(\operatorname{SOCC})_{a, s, k \mid k-1}+\hat{u}_{R Q, a, s, k \mid k-1}
$$

similar to (24) but using the estimated states SÔC and $\hat{u}_{R Q}$. Hereby, $\hat{i}_{p, k}$ is the estimated current, $\boldsymbol{K}_{p, k}^{s}$ is the Kalman gain, $\boldsymbol{P}_{p, k \mid k-1}^{s}$ is the predicted and $\boldsymbol{P}_{p, k \mid k}^{s}$ is the corrected estimation error covariance matrix, $\hat{\boldsymbol{x}}_{p, k \mid k-1}^{s}$ are the predicted and $\hat{\boldsymbol{x}}_{p, k \mid k}^{s}$ are the corrected states, $\boldsymbol{I}$ is the identity matrix, $\boldsymbol{R}_{p, k}^{s}=\mathrm{E}\left\{\boldsymbol{w}_{p, k}^{s} \boldsymbol{w}_{p, k}^{s, \top}\right\}$ is the measurement noise covariance matrix and $\boldsymbol{Q}_{p, k}^{s}=\mathrm{E}\left\{\boldsymbol{v}_{p, k}^{s} \boldsymbol{v}_{p, k}^{s, \top}\right\}$ is the system noise covariance matrix, and $\Upsilon_{p, \boldsymbol{\alpha}_{k}, j}^{s}$ is the extension of the binomial coefficient, for subsystem $p$. The nonlinearities of the system are treated by linearization. The required Jacobian matrices of the system and the output function are given by

$$
\begin{aligned}
\boldsymbol{F}_{p, k}^{s} & =\left[\frac{\partial \boldsymbol{f}_{p, k}^{s}\left(\boldsymbol{x}_{p, k}^{s}, i_{p, k}\right)}{\partial \boldsymbol{x}_{p, k}^{s}}\right]_{\boldsymbol{x}_{p, k}^{s}=\hat{\boldsymbol{x}}_{p, k \mid k}^{s}, i_{p, k}=\hat{i}_{p, k}} \\
\boldsymbol{G}_{p, k}^{s} & =\left[\frac{\partial \boldsymbol{g}_{p, k}^{s}\left(\boldsymbol{x}_{p, k}^{s}, i_{p, k}\right)}{\partial \boldsymbol{x}_{p, k}^{s}}\right]_{\boldsymbol{x}_{p, k}^{s}=\hat{\boldsymbol{x}}_{p, k \mid k-1}^{s}, i_{p, k}=\hat{i}_{p, k}} .
\end{aligned}
$$

As a result, the algorithm consists of two parts which cooperate with each other: the state estimation and the current estimation. This method needs the measurement of the cell voltages as well as the total voltage or the total current, only. Up to $P-1$ Sensors can be saved, because measurements of the single string currents are not necessary. Furthermore, due to the reduced order of the local string models and the local Kalman filtering, the complexity is decreased in comparison to a direct estimation of the pack model as discussed. Moreover, this approach is scalable because the battery pack and the distributed filters can be easily extended. 


\section{MEASUREMENT SETUP}

For the validation of the estimation algorithm and to evaluate the current estimation, an experiment has been set up. The setup contains three battery strings, with three KOKAM SLPB834374H lithium-ion cells per string. All individual cell voltages $y_{k}^{c}$ as well as the total voltage $u_{t, k}$ are measured using an A/D-board DS2004 from dSPACE. For control and measurement of the total battery current $i_{t, k}$ which is shown in Fig. 5, a BOP20-20M current source from Kepco is used. For comparison, each string contains also a highly accurate 34410A multimeter from Keysight Technologies to validate the estimated string currents. Since the currents $i_{m, p}$ are measured under laboratory conditions, they are further used as SOC-reference by a current integration. The additional resistances $R_{c, p}$ are assumed to be constant over time, but differ in each string and have been identified to

$$
R_{c, 1}=540 \mathrm{~m} \Omega, R_{c, 2}=610 \mathrm{~m} \Omega, R_{c, 3}=650 \mathrm{~m} \Omega .
$$

The initial states of the cells have been determined using voltage measurements of each cell after a rest time of $t=$ $1000 \mathrm{~s}$ and before wiring them to a pack. After the rest time it can be assumed that $u_{R Q, p, s}^{c} \approx 0 \mathrm{~V} \forall p, s$, so that the initial SOCs can be determined using the OCV-SOC relation. The determined initial states are

$$
\begin{aligned}
& \boldsymbol{x}_{1,0}^{s}=\left(\begin{array}{llllll}
95.3 \% & 0 \mathrm{~V} & 85.5 \% & 0 \mathrm{~V} & 74.9 \% & 0 \mathrm{~V}
\end{array}\right)^{\top}, \\
& \boldsymbol{x}_{2,0}^{s}=\left(\begin{array}{llllll}
90.5 \% & 0 \mathrm{~V} & 80.8 \% & 0 \mathrm{~V} & 69.4 \% & 0 \mathrm{~V}
\end{array}\right)^{\top}, \\
& \boldsymbol{x}_{3,0}^{s}=\left(\begin{array}{llllll}
85.6 \% & 0 \mathrm{~V} & 76.1 \% & 0 \mathrm{~V} & 64.6 \% & 0 \mathrm{~V}
\end{array}\right)^{\top} .
\end{aligned}
$$

Although, the cells may have different parameters in practice, it can not be expected that exact information about all cells is available. Therefore, the parameters $R_{i}, R, Q, \alpha, Q_{n}$ and the OCV-SOC relation have been identified only for one of the cells of the battery pack. Accordingly, we use the identified relationships between parameters and their particular SOC for all cells of the model.

\section{ESTIMATION RESULTS}

The measurements from Sec. V were used to validate the proposed algorithm. Based on the results of [15] and [29], we use a sampling time $T=0.1 \mathrm{~s}$, a memory length $L=250$,

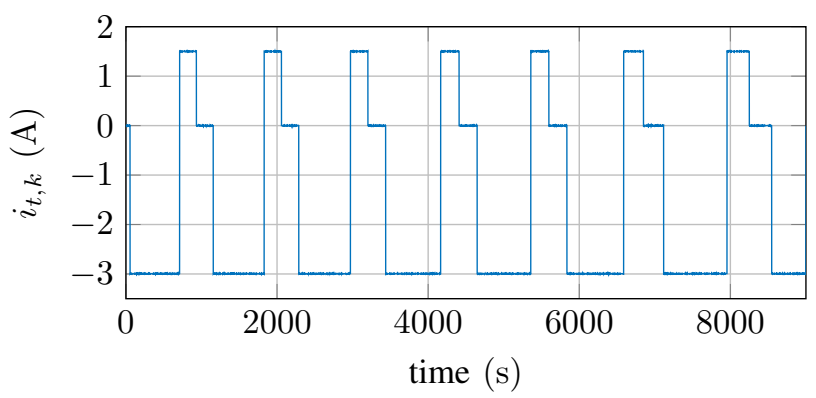

Fig. 5. Input current $i_{t, k}$ for the measurement setup. and covariance matrices

$$
\begin{aligned}
\boldsymbol{Q}_{p, k}^{s}= & \operatorname{diag}\left\{10^{-5} \%^{2}, 5 \cdot 10^{-4} \mathrm{~V}^{2}, 10^{-5} \%^{2},\right. \\
& \left.5 \cdot 10^{-4} \mathrm{~V}^{2}, 10^{-5} \%^{2}, 5 \cdot 10^{-4} \mathrm{~V}^{2}\right\} \quad \forall p, k \\
\boldsymbol{R}_{p, k}^{s}= & \operatorname{diag}\left\{2.8391 \cdot 10^{-8} \mathrm{~V}^{2}, 2.8391 \cdot 10^{-8} \mathrm{~V}^{2},\right. \\
& \left.2.8391 \cdot 10^{-8} \mathrm{~V}^{2}\right\} \quad \forall p, k \\
\boldsymbol{P}_{p, 0}^{s}= & \operatorname{diag}\left\{100 \%^{2}, 100 \mathrm{~V}^{2}, 100 \%{ }^{2},\right. \\
& \left.100 \mathrm{~V}^{2}, 100 \%^{2}, 100 \mathrm{~V}^{2}\right\} \quad \forall p .
\end{aligned}
$$

The initial states of the filter algorithm have been set to

$$
\begin{aligned}
\hat{\boldsymbol{x}}_{1,0}^{s} & =\left(\begin{array}{llllll}
93.3 \% & 0 \mathrm{~V} & 88.5 \% & 0 \mathrm{~V} & 79.9 \% & 0 \mathrm{~V}
\end{array}\right)^{\top}, \\
\hat{\boldsymbol{x}}_{2,0}^{s} & =\left(\begin{array}{llllll}
93.5 \% & 0 \mathrm{~V} & 77.8 \% & 0 \mathrm{~V} & 73.4 \% & 0 \mathrm{~V}
\end{array}\right)^{\top}, \\
\hat{\boldsymbol{x}}_{3,0}^{s} & =\left(\begin{array}{llllll}
81.6 \% & 0 \mathrm{~V} & 81.1 \% & 0 \mathrm{~V} & 63.6 \% & 0 \mathrm{~V}
\end{array}\right)^{\top}
\end{aligned}
$$

which have some randomly chosen deviations from the true states. The initial states are in practice unknown, so these deviations are used to check if the filters are able to handle initialization errors. The results of the experiment using case 1 (measurement of total current, communication between all subsystems) are shown in Tab. I and in Figs. 6 - 10. The rootmean-square error (RMSE) of the estimated currents is in the range of a few $\mathrm{mA}$ in this experiment as shown in Tab. I. The maximum absolute error (MAE) of the estimated currents is

TABLE I

SOC AND CURRENT ESTIMATION ERRORS

\begin{tabular}{c|c|c|c|c}
\hline String 1 & SOC Cell 1 & SOC Cell 2 & SOC Cell 3 & Current \\
RMSE & $1.7434 \%$ & $1.5595 \%$ & $1.7737 \%$ & $0.0126 \mathrm{~A}$ \\
MAE & $2.6492 \%$ & $2.9997 \%$ & $4.9994 \%$ & $1.4474 \mathrm{~A}$ \\
\hline String 2 & SOC Cell 1 & SOC Cell 2 & SOC Cell 3 & Current \\
RMSE & $0.5913 \%$ & $2.2826 \%$ & $1.5678 \%$ & $0.0095 \mathrm{~A}$ \\
MAE & $2.9996 \%$ & $3.4921 \%$ & $3.9997 \%$ & $1.3181 \mathrm{~A}$ \\
\hline String 3 & SOC Cell 1 & SOC Cell 2 & SOC Cell 3 & Current \\
RMSE & $1.1245 \%$ & $1.1602 \%$ & $2.0876 \%$ & $0.0094 \mathrm{~A}$ \\
MAE & $3.9996 \%$ & $4.9994 \%$ & $3.3015 \%$ & $1.2391 \mathrm{~A}$
\end{tabular}

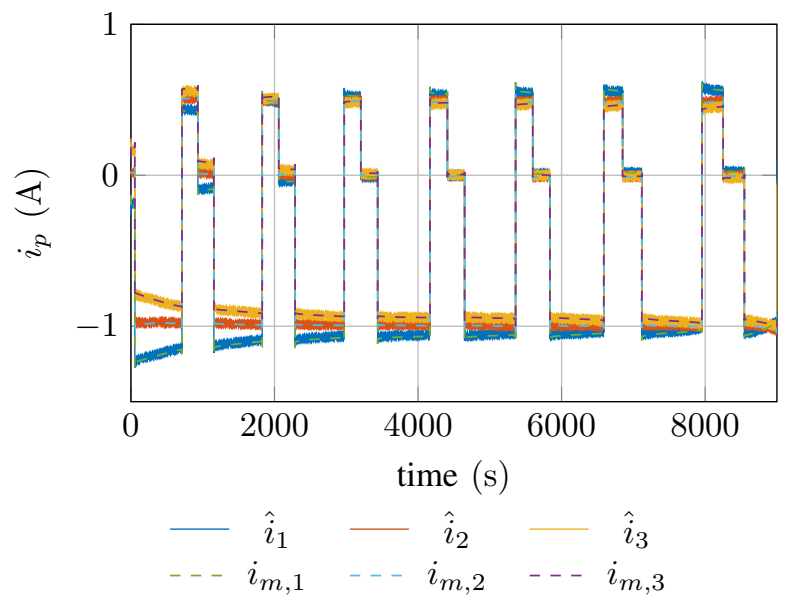

Fig. 6. Comparison of estimated string currents $\hat{i}_{p}$ and measured string currents $i_{m, p}$. 


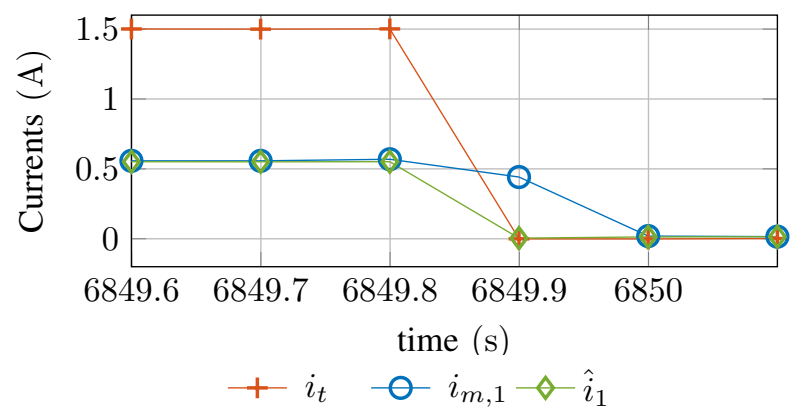

Fig. 7. Delay between measured total current $i_{t}$ and measured string current $i_{m, 1}$ with induced difference to estimated current $\hat{i}_{1}$.

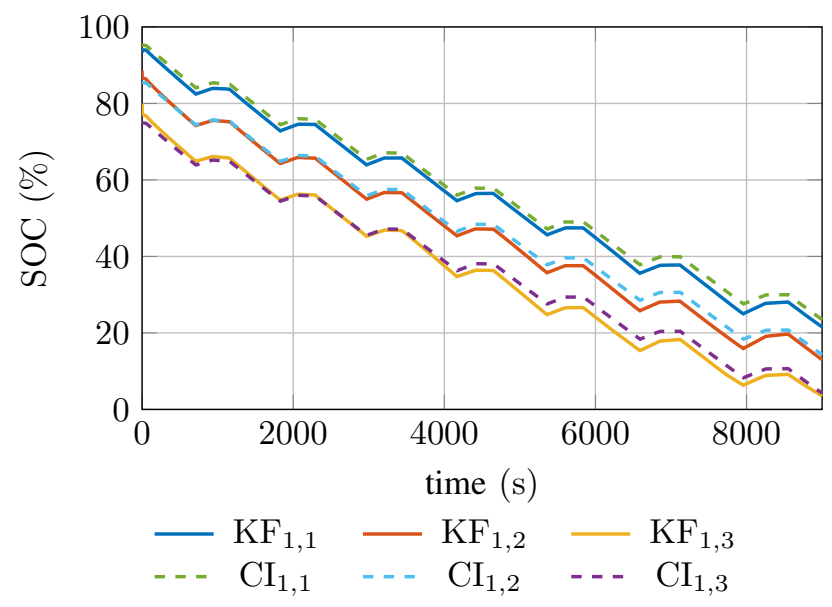

Fig. 8. SOCs estimated by the DFEKF (KF) of string 1 and SOCs calculated by a current integration (CI) of measured current $i_{m, 1}$.

larger, but only due to different jitter periods and time delays of the measurement equipment around the current steps of $i_{t}$ and therefore, they are not informative. An exemplary excerpt of a current step is shown in Fig. 7.

Fig. 6 shows the estimated string currents $\hat{i}_{p}$ by the DFEKF as well as the measured currents $i_{m, p}$ by the Keysight multimeters. It can be seen that there are only small differences between these currents. The remaining errors are induced by measurement noises of the total current and the cell voltages. As a result, the proposed current estimation procedure can compete with the highly accurate multimeters.

In Figs. 8 and 9 the estimated SOCs of the DFEKF are shown for all cells of strings 1 and 2 as well as the corresponding SOCs which are calculated by a current integration using the measured branch currents $i_{m, p}$. Fig. 10, however, shows exemplary the error between the estimated SOCs and the SOCs determined by the current integration of string 3. The RMSE and MAE of all cells are also shown in Tab. I. The SOC estimation error is at all times for all strings smaller than $5 \%$. For most of the cells the MAE is equal to the initial SOC error. Only three cells exceed it. The results match with the conclusions for single-cell estimation of [15] which stated that the largest errors occur in the flat region of the OCV-SOC curve. The states can be estimated accurately even though the filters are not correctly initialized

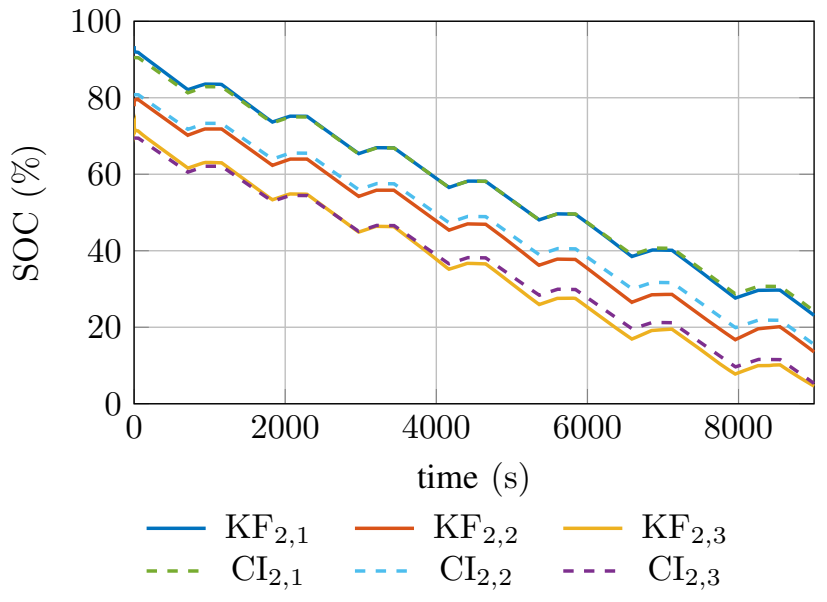

Fig. 9. SOCs estimated by the DFEKF (KF) of string 2 and SOCs calculated by a current integration (CI) of measured current $i_{m, 2}$.

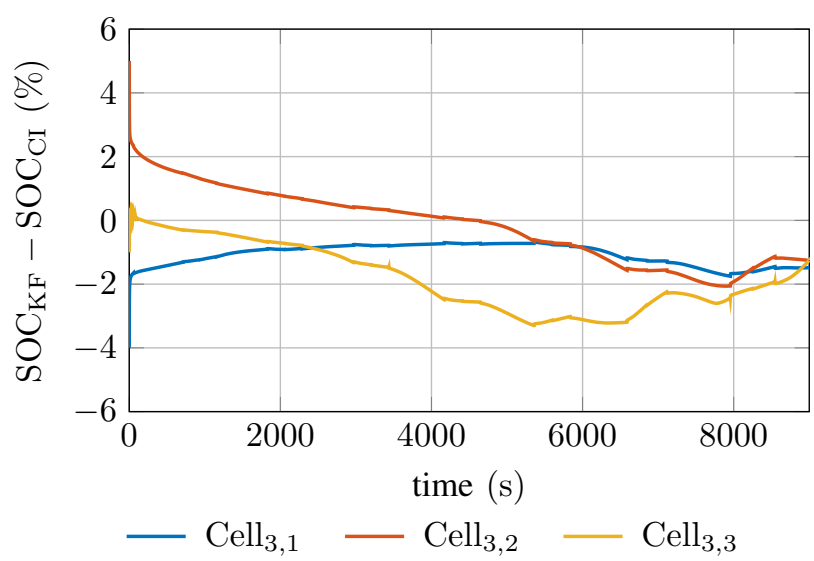

Fig. 10. Estimation error between estimated SOCs by the DFEKF of string 3 and SOCs calculated by a current integration of measured current $i_{m, 3}$.

and cell-to-cell parameter variations have been disregarded.

We also proceeded the proposed method for case 2 (measurement of total voltage, no communication). The results are not illustrated because they are nearly identical to case 1 except some very small variations due to slightly different measurement noises. Hence, the second case provides identical results, although communication between the subsystems is not needed.

\section{SUMMARY AND CONCLUSION}

In this paper, a distributed fractional extended Kalman filter has been proposed for state of charge and current estimation of lithium-ion battery packs. Based on a fractional 1-RQ model of a battery cell, the model of a battery pack has been derived. In order to minimize measuring effort, two cases for a model based calculation of the current distribution has been proposed and used for string current estimation. Since the only dependency between the battery strings is described by the current calculation model, a string-wise distribution of the system into subsystems could be enabled. Each subsystem estimates only the states of its own string and is therefore of reduced order compared to the battery 
pack. Measurement results of a battery pack consisting of three strings with three cells, each, show that the proposed method is suitable for SOC as well as current estimations. Depending on available measurements, the subsystems do not need to communicate with each other (case 2).

In contrast to existing literature, the proposed approach is capable of a model based estimation of the string currents which attains reduced measurement effort. Apart from accurate estimation results, an important feature of the presented approach is its versatility. That is, the distribution of the total battery pack is not mandatory for the current estimation procedure, but achieves advantages like increased scalability and smaller subsystems with reduced order. Furthermore, the cell or pack model can be exchanged straightforwardly using more complex models, or alternatively, using simple integerorder models with $\alpha=1$ and omitting fractional calculus. Future works are the extension of the algorithm by an online identification of the battery resistances.

\section{REFERENCES}

[1] L. Lu, X. Han, J. Li, J. Hua, and M. Ouyang, "A review on the key issues for lithium-ion battery management in electric vehicles," Journal of Power Sources, vol. 226, pp. 272-288, 2013.

[2] M.-H. Chang, H.-P. Huang, and S.-W. Chang, "A new state of charge estimation method for LiFePO4 battery packs used in robots," Energies, vol. 6, no. 4, pp. 2007-2030, 2013.

[3] A. Fotouhi, D. J. Auger, K. Propp, S. Longo, and M. Wild, "A review on electric vehicle battery modelling: From lithium-ion toward lithium-sulphur," Renewable and Sustainable Energy Reviews, vol. 56, pp. 1008-1021, 2016.

[4] Y. He, X. Liu, C. Zhang, and Z. Chen, "A new model for stateof-charge (SOC) estimation for high-power Li-ion batteries," Applied Energy, vol. 101, pp. 808-814, 2013.

[5] W.-Y. Chang, "The state of charge estimating methods for battery: A review," ISRN Applied Mathematics, vol. 2013, 2013.

[6] W. Waag, C. Fleischer, and D. U. Sauer, "Critical review of the methods for monitoring of lithium-ion batteries in electric and hybrid vehicles," Journal of Power Sources, vol. 258, pp. 321-339, 2014.

[7] K. S. Ng, C.-S. Moo, Y.-P. Chen, and Y.-C. Hsieh, "Enhanced coulomb counting method for estimating state-of-charge and state-of-health of lithium-ion batteries," Applied Energy, vol. 86, no. 9, pp. 1506-1511, 2009.

[8] K. A. Smith, C. D. Rahn, and C.-Y. Wang, "Control oriented 1d electrochemical model of lithium ion battery," Energy Conversion and Management, vol. 48, no. 9, pp. 2565-2578, 2007.

[9] M. Charkhgard and M. Farrokhi, "State-of-charge estimation for lithium-ion batteries using neural networks and EKF," IEEE Transactions on Industrial Electronics, vol. 57, no. 12, pp. 4178-4187, 2010.

[10] J. Sabatier, M. Merveillaut, J. M. Francisco, F. Guillemard, and D. Porcelatto, "Lithium-ion batteries modeling involving fractional differentiation," Journal of Power Sources, vol. 262, pp. 36-43, 2014.

[11] B. Wang, S. E. Li, H. Peng, and Z. Liu, "Fractional-order modeling and parameter identification for lithium-ion batteries," Journal of Power Sources, vol. 293, pp. 151-161, 2015.

[12] Y. Ma, X. Zhou, B. Li, and H. Chen, "Fractional modeling and SOC estimation of lithium-ion battery," IEEE/CAA Journal of Automatica Sinica, vol. 3, no. 3, pp. 281-287, 2016.

[13] B. Wang, Z. Liu, S. E. Li, S. J. Moura, and H. Peng, "State-of-charge estimation for lithium-ion batteries based on a nonlinear fractional model," IEEE Transactions on Control Systems Technology, vol. 25, no. 1, pp. 3-11, 2017.

[14] R. Xiao, J. Shen, X. Li, W. Yan, E. Pan, and Z. Chen, "Comparisons of modeling and state of charge estimation for lithium-ion battery based on fractional order and integral order methods," Energies, vol. 9, no. 3, p. 184, Mar. 2016.

[15] M. Kupper, C. Funk, M. Eckert, and S. Hohmann, "Fractional extended and unscented Kalman filtering for state of charge estimation of lithium-ion batteries," in 2018 Annual American Control Conference (ACC). IEEE, 2018, pp. 3855-3862.
[16] F. Sun and R. Xiong, "A novel dual-scale cell state-of-charge estimation approach for series-connected battery pack used in electric vehicles," Journal of Power Sources, vol. 274, pp. 582-594, 2015.

[17] H. Dai, Z. Sun, and X. Wei, "Online SOC estimation of highpower lithium-ion batteries used on HEVs," in IEEE International Conference on Vehicular Electronics and Safety. ICVES 2006. IEEE, 2006, pp. 342-347.

[18] H. Dai, X. Wei, and Z. Sun, "State and parameter estimation of a HEV Li-ion battery pack using adaptive Kalman filter with a new SOCOCV concept," in International Conference on Measuring Technology and Mechatronics Automation. ICMTMA'09, vol. 2. IEEE, 2009, pp. 375-380.

[19] H. Li, C. Liao, and L. Wang, "Research on state-of-charge estimation of battery pack used on hybrid electric vehicle," in Asia-Pacific Power and Energy Engineering Conference. APPEEC 2009. IEEE, 2009, pp. $1-4$.

[20] C. Zhang, J. Jiang, W. Zhang, and S. M. Sharkh, "Estimation of state of charge of lithium-ion batteries used in HEV using robust extended Kalman filtering," Energies, vol. 5, no. 4, pp. 1098-1115, 2012.

[21] G. L. Plett, "Efficient battery pack state estimation using bar-delta filtering," in EVS24 international battery, hybrid and fuel cell electric vehicle symposium, 2009, pp. 1-8.

[22] H. Dai, X. Wei, Z. Sun, J. Wang, and W. Gu, "Online cell SOC estimation of Li-ion battery packs using a dual time-scale Kalman filtering for EV applications," Applied Energy, vol. 95, pp. 227-237, 2012.

[23] R. Xiong, F. Sun, X. Gong, and H. He, "Adaptive state of charge estimator for lithium-ion cells series battery pack in electric vehicles," Journal of Power Sources, vol. 242, pp. 699-713, 2013.

[24] T. Bruen and J. Marco, "Modelling and experimental evaluation of parallel connected lithium ion cells for an electric vehicle battery system," Journal of Power Sources, vol. 310, pp. 91-101, 2016.

[25] N. Yang, X. Zhang, B. Shang, and G. Li, "Unbalanced discharging and aging due to temperature differences among the cells in a lithium-ion battery pack with parallel combination," Journal of Power Sources, vol. 306, pp. 733-741, 2016.

[26] S. Sepasi, R. Ghorbani, and B. Y. Liaw, "Improved extended Kalman filter for state of charge estimation of battery pack," Journal of Power Sources, vol. 255, pp. 368-376, 2014.

[27] C. Y. Chun, J. Baek, G.-S. Seo, B. Cho, J. Kim, I. K. Chang, and S. Lee, "Current sensor-less state-of-charge estimation algorithm for lithium-ion batteries utilizing filtered terminal voltage," Journal of Power Sources, vol. 273, pp. 255-263, 2015.

[28] M.-S. Wu, C.-Y. Lin, Y.-Y. Wang, C.-C. Wan, and C. Yang, "Numerical simulation for the discharge behaviors of batteries in series and/or parallel-connected battery pack," Electrochimica Acta, vol. 52, no. 3, pp. 1349-1357, 2006.

[29] M. Kupper, J. Brenneisen, O. Stark, S. Krebs, and S. Hohmann, "Cascaded fractional Kalman filtering for state and current estimation of large-scale lithium-ion battery packs," in 2018 Chinese Control And Decision Conference (CCDC). IEEE, 2018, pp. 5071-5078.

[30] N. Tian, H. Fang, and Y. Wang, "3-d temperature field reconstruction for a lithium-ion battery pack: A distributed kalman filtering approach," IEEE Transactions on Control Systems Technology, no. 99, pp. 1-8, 2017.

[31] C. A. Monje, Y. Chen, B. M. Vinagre, D. Xue, and V. Feliu-Batlle, Fractional-order systems and controls: fundamentals and applications. Springer Science \& Business Media, 2010.

[32] D. Valério and J. Sá da Costa, "Variable order fractional controllers," Asian Journal of Control, vol. 15, no. 3, pp. 648-657, 2013.

[33] I. Podlubny, Fractional differential equations: an introduction to fractional derivatives, fractional differential equations, to methods of their solution and some of their applications. Elsevier, 1998, vol. 198.

[34] J. Sabatier, C. Farges, and J.-C. Trigeassou, "Fractional systems state space description: some wrong ideas and proposed solutions," Journal of Vibration and Control, vol. 20, no. 7, pp. 1076-1084, 2014.

[35] M. Eckert, M. Kupper, and S. Hohmann, "Functional fractional calculus for system identification of battery cells," atAutomatisierungstechnik, vol. 62, no. 4, pp. 272-281, 2014.

[36] M. Kupper, I. S. Gil, and S. Hohmann, "Distributed and decentralized state estimation of fractional order systems," in American Control Conference (ACC), 2016. IEEE, 2016, pp. 2765-2771. 